\title{
Application of DLVO Energy Map To Evaluate Interactions between Spherical Colloids and Rough Surfaces
}

\author{
Chongyang Shen, ${ }^{* \dagger}{ }^{\dagger}$ Feng Wang, ${ }^{\ddagger}$ Baoguo Li, ${ }^{\dagger}$ Yan Jin, ${ }^{\S}$ Lian-Ping Wang, ${ }^{\perp}$ and Yuanfang Huang ${ }^{*}, \dagger$ \\ ${ }^{\dagger}$ Department of Soil and Water Sciences, China Agricultural University, Beijing 100193, China \\ ${ }^{\ddagger}$ Institute of Desertification, Chinese Academy of Forestry, Beijing 100910, China \\ ${ }^{\S}$ Department of Plant and Soil Sciences, University of Delaware, Newark, Delaware 19716, United States \\ ${ }^{\perp}$ Department of Mechanical Engineering, University of Delaware, Newark, Delaware 19716, United States
}

\section{Supporting Information}

\begin{abstract}
This study theoretically evaluated interactions between spherical colloids and rough surfaces in three-dimensional space using Derjaguin-LandauVerwey- Overbeek (DLVO) energy/force map and curve. The rough surfaces were modeled as a flat surface covered by hemispherical protrusions. A modified Derjaguin approach was employed to calculate the interaction energies and forces. Results show that more irreversible attachments in primary minima occur at higher ionic strengths, which theoretically explains the observed hysteresis of colloid attachment and detachment during transients in solution chemistry. Secondary minimum depths can be increased significantly in concave regions (e.g., areas aside of asperities or between asperities) due to sidewall interactions. Through comparing the tangential attractive forces from asperities and the hydrodynamic drag forces in three-dimensional space, we showed that attachment in secondary minima can be located on open collector surfaces of a porous medium. This result challenges the usual belief that the

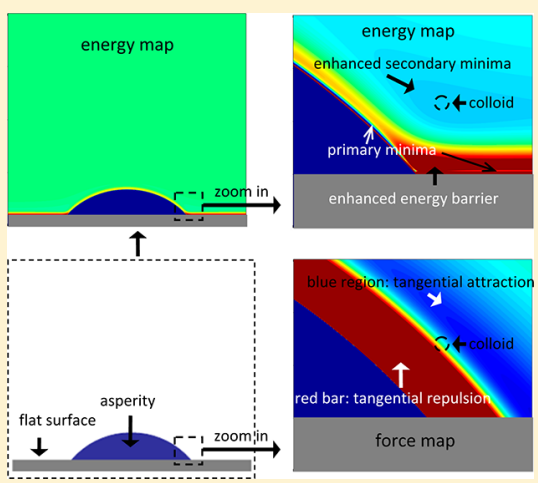
attachment in secondary minima only occurs in stagnation point regions of the porous medium and is absent in shear flow systems such as parallel plate flow chamber and impinging jet apparatus. Despite the argument about the role of secondary minima in colloid attachment remained, our study theoretically justified the existence of attachment in secondary minima in the presence of surface roughness. Further, our study implied that the presence of surface roughness is more favorable for attachment in secondary minima than in primary minima under unfavorable chemical conditions.
\end{abstract}

\section{INTRODUCTION}

Knowledge of the interaction energy between a colloid and a collector surface is of primary importance in prediction of colloid attachment behavior in porous media. The theoretical framework for determining the colloid-collector interaction energy is provided by a landmark theory developed by Derjaguin and Landau ${ }^{1}$ and Verwey and Overbeek ${ }^{2}$ (i.e., DLVO theory). The interaction energy curve is frequently adopted in this theory to quantitatively describe the colloidcollector interaction energy including van der Waals attraction (VDW) and double layer energy (DL). ${ }^{3-5}$ The energy curve is commonly constructed by summing the VDW and DL over the surface-to-surface separation distance between the colloid and collector. When both colloid and collector surfaces are likecharged, a typical potential energy curve is characterized by an infinitely deep attractive well (i.e., primary minimum) at a small separation distance, a maximum energy barrier, and an attractive well (i.e., secondary minimum) at larger distances. Both energy barrier and secondary minimum disappear and only primary minimum exist in the DLVO interaction energy curve if the colloid and collector surfaces are oppositely charged. The depth of primary minimum is finite if short-range repulsion (e.g., hydration and steric repulsion) is included in the total potential energy. ${ }^{6}$

The use of interaction energy curve greatly assists evaluation of colloid transport behavior. For example, a large energy barrier indicates that attachment in primary minima is impossible, while a deep secondary minimum illustrates its ability to capture colloids. However, it should be mentioned that the interaction energy curves with aforementioned shapes are obtained by calculating the interaction energy in one dimension. When both colloid and collector have ideal surface properties (e.g., physically smooth and chemically homogeneous surface), only one interaction energy curve is capable of describing the interaction energy of the colloid with the collector surface. However, if surface heterogeneity (e.g., roughness) is present, the interaction energy curve varies from place to place on the collector surface. Hence, it is difficult to completely characterize the colloid-collector interaction through using the energy curve. Recently, Hoek et al. ${ }^{7}$ and Kemps and Bhattacharjee ${ }^{8}$ presented a new technique, i.e.,

Received: August 4, 2012

Revised: September 24, 2012

Published: September 24, 2012 
interaction energy map, to describe the interactions between colloids and rough surfaces. The interaction energy map has advantage over the energy curve to describe the colloidcollector surface interaction in the presence of surface morphological heterogeneity. ${ }^{7,8}$

The objective of this study was to theoretically examine the DLVO interaction energies between spherical colloids and rough surfaces in three-dimensional space using both interaction energy map and curve. Spatial attention was paid to the interaction of a colloid with its lateral asperities (i.e., sidewall interaction) that has been ignored in existing literature. $^{7-12}$ A modified Derjaguin approach was adopted to calculate the DLVO interaction energies. We showed that more irreversible attachments in primary minima occur at higher ionic strengths. We highlighted that the presence of surface roughness favors colloid attachment at secondary minima in concave regions where the secondary minimum energy is increased and hydrodynamic shear is decreased. Whereas colloid attachment in secondary minima is commonly regarded to occur only in stagnation point regions of a porous medium, results of this study show that the attachment can be located on open collector surfaces in the porous medium.

\section{THEORETICAL CONSIDERATIONS}

2.1. Model of Rough Surfaces. Similar to previous studies, ${ }^{7,9-18}$ surface roughness was modeled as hemispherical asperities on flat surfaces. We employed three elemental rough models: a hemisphere on a flat surface and two or three hemispheres against each other on a flat surface (see Figure 1). These elemental models were used to represent two typical rough configurations according to atomic force microscopy (AFM) examinations: ${ }^{7,13,14,19,20}$ convex asperities and concave valleys aside and between the asperities.

2.2. Calculation of DLVO Interaction Energy in ThreeDimensional Space. Surface element integration (SEI) has been frequently used to calculate DLVO interaction energies between colloids and rough surfaces. ${ }^{7-11,21,22}$ The technique provides exact evaluation of the interaction energy provided that one of the interaction surfaces is flat. ${ }^{21}$ Thus, the SEI has made a remarkable breakthrough in the interaction energy calculation based on Derjaguin's approximation. However, there is an assumption in the SEI if it is used for the case where both interacting surfaces are curved. Specifically, the SEI has to take the interaction of a colloid surface element with the entire of real collector surface same as the interaction of the element with an infinite flat plate parallel to it. As such, the developed analytical equations used to exactly calculate the $\mathrm{VDW}^{23}$ and $\mathrm{DL}^{24}$ for the interaction of an element with an infinite flat plate parallel to it can be utilized by the SEI. Such treatment causes discrepancies between the true interaction energies and the calculated results for colloid-rough surface interaction. For example, there is a limitation in the SEI that it only considers the interactions with the rough surface area that overlaps with the projected area of an approaching colloid. ${ }^{9}$ Hence, the SEI underestimates interaction energies if sidewall interactions are significant. Details about why the SEI cannot accurately estimate colloid-rough surface interaction energy are given in the Supporting Information. On the basis of the SEI, Duffadar and Davis ${ }^{25}$ and Bendersky and Davis ${ }^{22}$ developed grid-surface integration (GSI) to calculate DLVO interaction energy in the presence of both surface roughness and charge heterogeneity. The only difference between the SEI and GSI is that the SEI discretizes colloid surface whereas the GSI discretizes collector

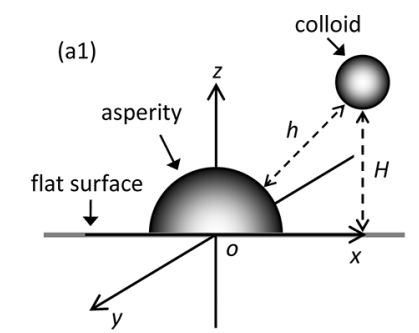

(a2)
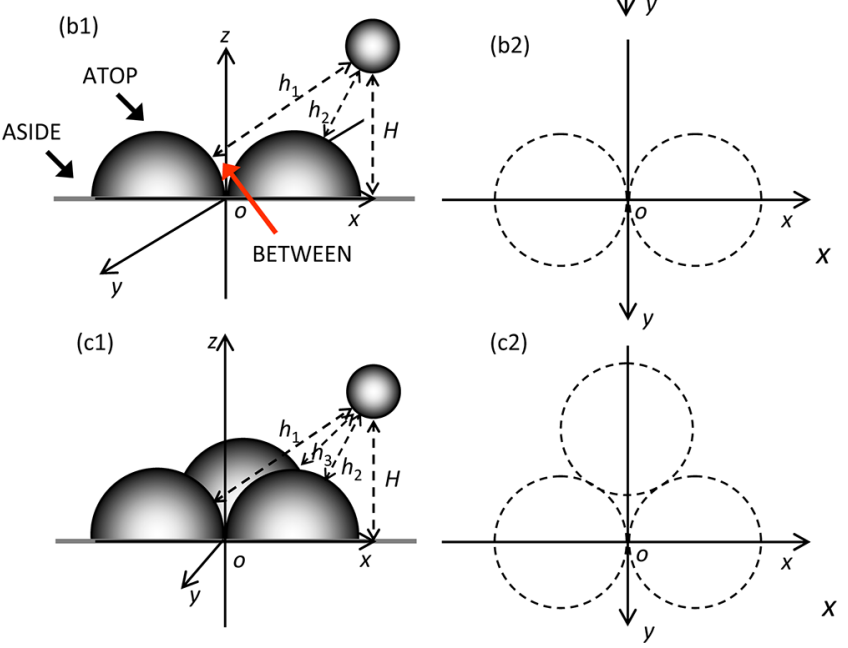

Figure 1. Coordinate systems used to describe a spherical particle interacting with three elemental rough models: (a) a hemisphere on a flat surface; (b) two hemispheres against each other on a flat surface; (c) three hemispheres against each other on a flat surface. Parts (a2), (b2), and (c2) are plan view images of parts (a1), (b1), and (c1), respectively. ATOP, ASIDE, and BETWEEN represent regions atop of asperities, aside asperities, and between asperities. Modified from Shen et al. $^{27}$

surface. Therefore, the GSI does not eliminate the aforementioned limitations in the SEI and also cannot accurately estimate the interaction energy between a colloid and rough surface.

Similar to previous studies, ${ }^{10,11,13-15,17,18,26}$ the interaction energies between a colloid and the rough surfaces in Figure 1 were determined as a sum of particle-surface (sphere-plate) and particle-asperity (sphere-sphere) DLVO interaction energies. The Cartesian coordinate system was used to determine the surface-to-surface separation distances between the colloid and the plate/asperity in three-dimensional space. For interaction of a colloid with a hemisphere on a flat surface (Figure 1a), the center of the hemisphere is assigned as the origin and the bottom of the hemisphere is located on the $x-y$ plane. For the interaction of a colloid with two hemispheres on a flat surface, the contact point of the two hemispheres is defined as the origin of the coordinate system, and the bottoms of the two hemispheres are located on the $x-y$ plane. The centers of the two hemispheres are assigned coordinates $\left(-a_{\mathrm{g}}\right.$ $0,0)$ and $\left(a_{\mathrm{g}}, 0,0\right)$, respectively, where $a_{\mathrm{g}}$ is asperity radius. For the interaction of a colloid with three hemispheres on a flat surface, a contact point of any two hemispheres is taken as the origin of the coordinate system, and the bottoms of the three hemispheres are located on the $x-y$ plane. The centers of the three hemispheres are assigned coordinates $\left(-a_{\mathrm{g}}, 0,0\right),\left(a_{\mathrm{g}}, 0\right.$, $0)$, and $\left(0,-\sqrt{ } 3 a_{\mathrm{g}}, 0\right)$, respectively. The center of the colloid is assigned coordinates $(x, y, z)$. The separation distances between the colloid and a hemisphere $(h)$ or the flat surface 

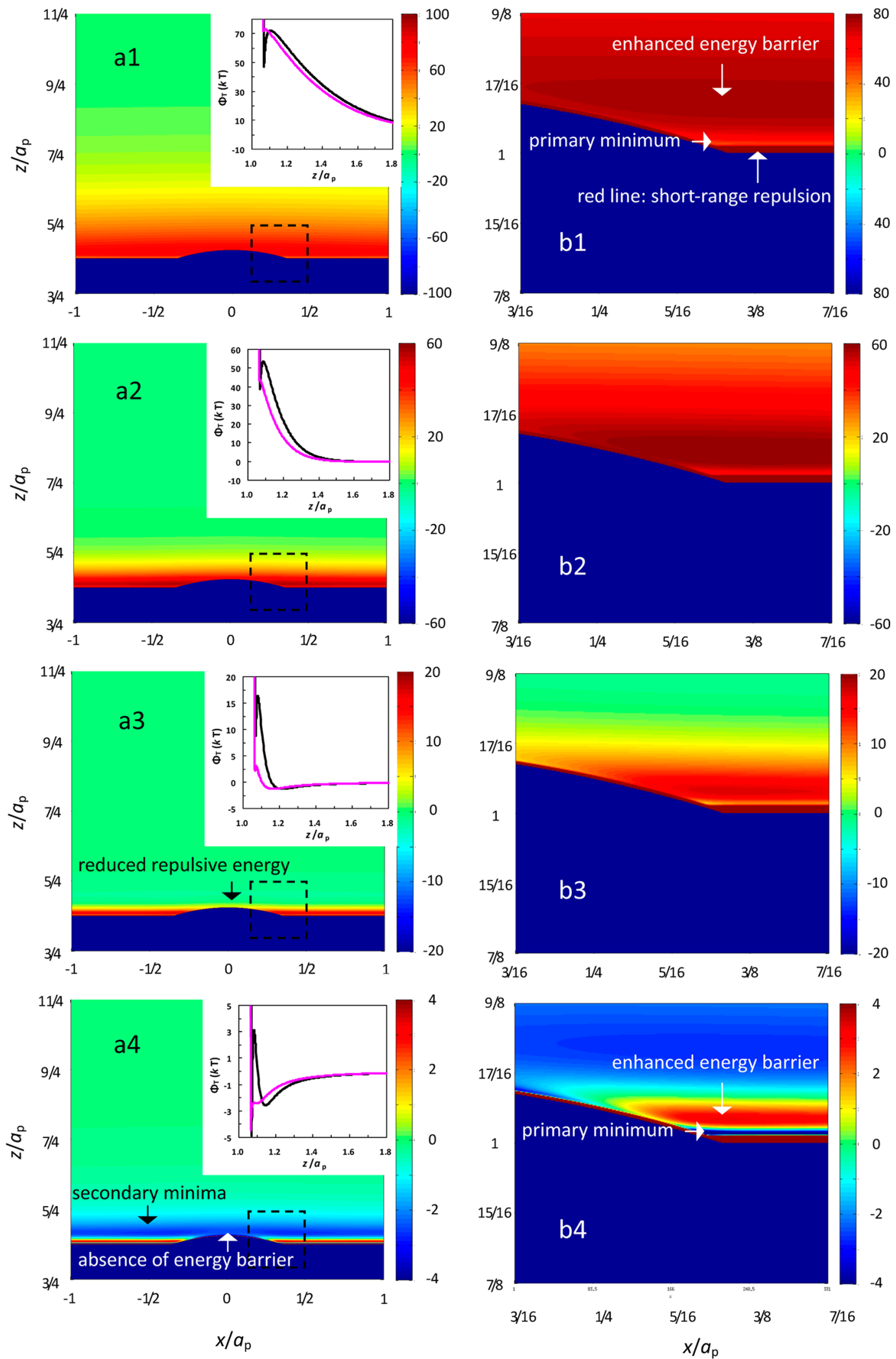

Figure 2. DLVO energy maps for interactions between a $66 \mathrm{~nm}$ colloid and the rough configuration in Figure 1a of $2 \mathrm{~nm}$ asperity radius on the $x-z$ plane at $y=0$ at different ionic strengths $(1,0.001 \mathrm{M} ; 2,0.01 \mathrm{M} ; 3,0.1 \mathrm{M} ; 4,0.2 \mathrm{M})$. (b) Zoomed maps for dashed-square regions in (a). The scale bar to the right of each plot represents the interaction energy in units of $k T$. The dark blue regions below the red lines indicate the locations where the interaction energy could not be determined due to physical overlap between the colloid and rough surface. Insets are DLVO energy curves along the $z$-axis of the coordination system in Figure 1a (pink line) and for interactions of the colloid with a flat surface (dark line).

$(H)$ for the three rough models are given in Table S1 of the Supporting Information.
The particle-asperity or particle-flat surface interaction energies were calculated by summing van der Waals attraction, 

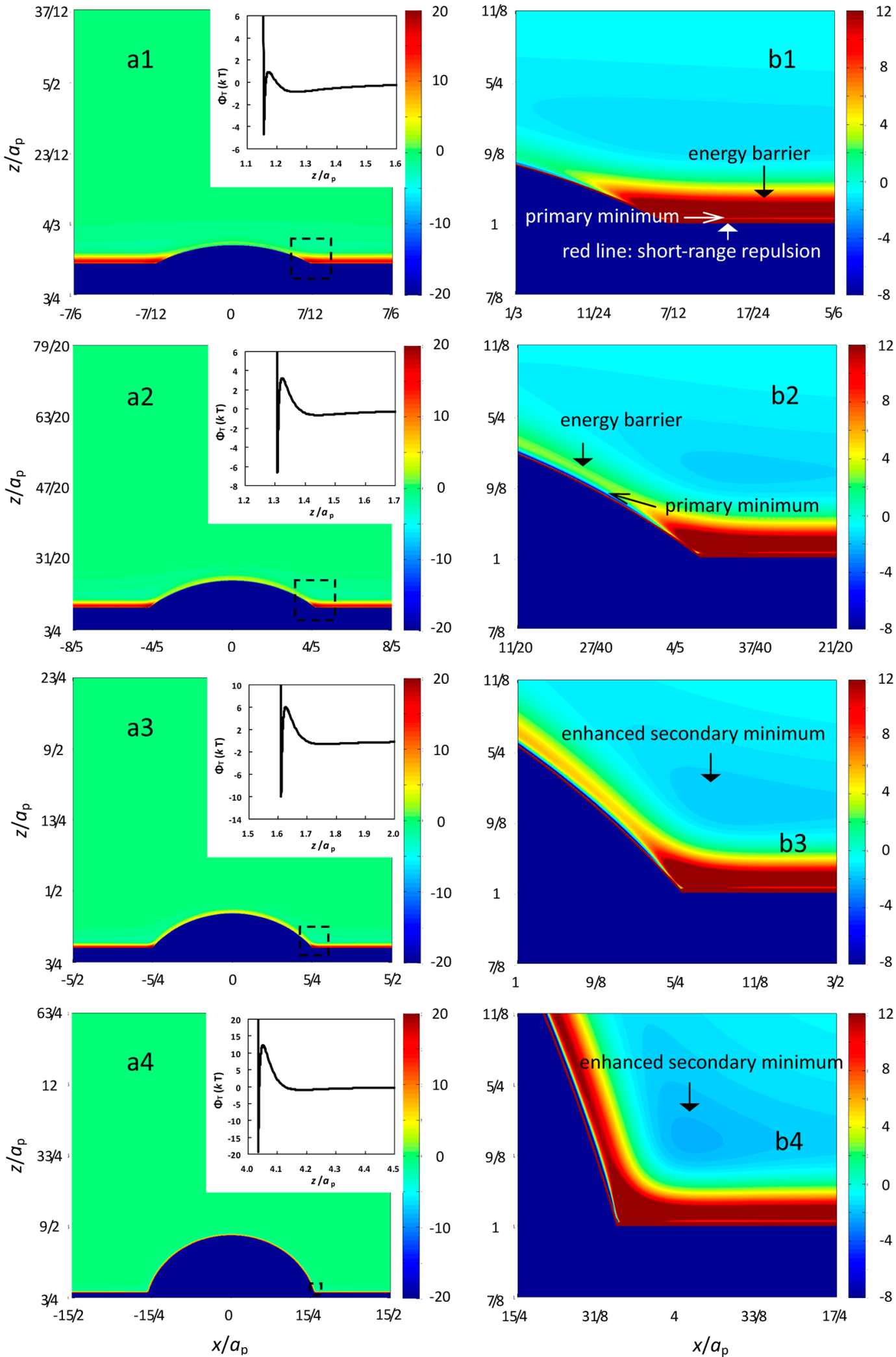

Figure 3. DLVO energy maps for interactions between the $66 \mathrm{~nm}$ colloid and rough configuration in Figure 1 a on the $x-z$ plane at $y=0$ at $0.1 \mathrm{M}$ ionic strength with different asperity radii $(1,5 \mathrm{~nm} ; 2,10 \mathrm{~nm} ; 3,20 \mathrm{~nm} ; 4,100 \mathrm{~nm})$. All simulation conditions were consistent with those in Figure 2 except the interaction energy scales. Insets are DLVO energy curves along the $z$-axis of the coordination system in Figure la.

double layer interaction energy, and short-range repulsion. Details about the equations used to calculate the particle-plate and particle-asperity interaction energies can be referred to Shen et al. ${ }^{14,27}$ A Matlab program was developed to implement the calculations in three-dimensional space. In the superposition treatment, there is certain level of repetition. Shen et al., ${ }^{14}$ however, showed that the repetition does not have significant influence on calculated results because the 
interaction energy comes mainly from a small region around the point of closest approach distance according to the Derjaguin's approximation. $1,2,21$

\section{RESULTS}

For theoretical calculations, we assumed the colloids and collectors to be polystyrene latex microspheres (with diameters of 66 and $1156 \mathrm{~nm}$ ) and sand grain, respectively, having the same properties as those used in Shen et al. ${ }^{2628}$ The DLVO interaction energies were calculated for the colloids in $\mathrm{NaCl}$ solutions with different ionic strengths $(0.001,0.01,0.1$, and 0.2 $\mathrm{M})$ at a $\mathrm{pH}$ value of 10 . The zeta potentials of both colloids and sand over the range of ionic strengths were adopted from the study of Shen et al. ${ }^{26,28}$ and shown in Table S2 of the Supporting Information. A value of $1 \times 10^{-20} \mathrm{~J}$ was chosen as the Hamaker constant for the polystyrene-water-quartz system. $^{13,14,17,18,28}$

3.1. Impact of Surface Roughness on Attachment in Primary Minima. Figure 2 presents DLVO energy maps for interactions between the $66 \mathrm{~nm}$ colloid and rough configuration in Figure 1a of $2 \mathrm{~nm}$ asperity radius on the $x-z$ plane at 0.001 , $0.01,0.1$, and $0.2 \mathrm{M}$. These energy maps were obtained by varying the vertical positions $(x, z)$ of the colloid center in a rasterized manner. The scale bar to the right of each plot represents the interaction energy in units of $k T$ ( $k$ is Boltzmann constant and $T$ is absolute temperature). The red line represents the short-range repulsion. The dark blue regions below the red lines indicate the locations where the interaction energy could not be determined due to physical overlap between the colloid and rough surfaces; therefore, the interaction energies in these regions were ignored. Figure 2 shows that the interaction energy barriers are reduced in regions atop of the asperity (denoted as ATOP) whereas enhanced in areas aside the asperity (denoted as ASIDE). Particularly, the energy barriers completely disappear at $0.2 \mathrm{M}$ in the ATOP. However, large energy barriers ( $>40 \mathrm{kTs}$, mapped in red) still exist in the ATOP at 0.01 and $0.001 \mathrm{M}$. The Boltzmann factor model $[\alpha=\exp (-\Delta \Phi)$, where $\alpha$ is attachment efficiency and $\Delta \Phi$ is energy barrier] shows that only several $k T$ s of energy barrier can essentially restrain colloids from being attached in primary minimum. Thus, only at high solution ionic strengths can the energy barriers be reduced enough to cause the colloids to be attached in the ATOP at primary minimum.

The insets in Figure 2 present the interaction energy curves along $z$-axis of the coordinate system in Figure 1a. The energy curves for interactions of the $66 \mathrm{~nm}$ colloid with a flat surface are also shown for comparison. Although the maximum energy barriers are not evidently reduced at ionic strengths of 0.001 and $0.01 \mathrm{M}$ by the presence of the $2 \mathrm{~nm}$ asperity, the primary minimum depths (or detachment energy barrier from primary minimum, obtained by subtracting primary minimum from maximum energy barrier) are greatly lowered. Specifically, the calculated primary minimum depths are 24.83 and $14.63 \mathrm{kT}$ at 0.001 and $0.01 \mathrm{M}$ for the interaction between the $66 \mathrm{~nm}$ colloid and flat surface, respectively. The depths are reduced to be only 1.85 and $0.4 k T$ at 0.001 and $0.01 \mathrm{M}$ for the interaction between the $66 \mathrm{~nm}$ colloid and rough surface in the $z$-direction, respectively. The depths are close to or smaller than the average kinetic energy of a colloid (i.e., $1.5 \mathrm{kT}$ ). Therefore, although the colloid is favored to be attached atop of the asperity in primary minima at high ionic strengths (e.g., at $0.2 \mathrm{M}$ ), the attached colloid will detach from primary minima by Brownian motion upon reduction of solution ionic strength. A number of column studies ${ }^{14,27,29}$ have shown that attachment in primary minima is reversible upon reduction of solution ionic strength. The low primary minimum depths or absence of primary minima are very common in AFM approach curves under unfavorable conditions. $^{30-34}$

Figure 3 presents DLVO energy maps for interactions of the $66 \mathrm{~nm}$ colloid with the rough surface in Figure 1a on the $x-z$ plane at $0.1 \mathrm{M}$ for different asperity radii. The energy curves along the $z$-axis are also shown in the insets. The energy barrier in the ATOP increases with increasing asperity radius from 5 to $100 \mathrm{~nm}$. However, comparison of Figure 3(a1) with Figure 2(a3) shows that the energy barrier in the ATOP decreases when the asperity radius increases from 2 to $5 \mathrm{~nm}$. Therefore, at a given ionic strength, there exists a critical value of asperity radius for a given colloid where the energy barrier in the ATOP reach a minimum, below and above which it increases. This is consistent with the results in Henry et al. ${ }^{12}$ The DLVO energy curves along the $z$-axis in Figure 2(a3) and in Figure 3 show that when $2 \mathrm{~nm} \leq a_{\mathrm{g}} \leq 10 \mathrm{~nm}$, the reduced energy barriers in the ATOP are small enough $(<3.2 k T)$ for the colloid to overcome by Brownian motion and attach in primary minima.

Figure 4 presents DLVO interaction energy curves for interactions between the $66 \mathrm{~nm}$ colloid and rough surface in Figure 1a of different asperity radii along the $z$-axis at different ionic strengths. The energy barriers are smaller than $5 \mathrm{kT}$ at 0.2 $\mathrm{M}$ for all asperity radii considered. Particularly, the energy barriers disappear for $2 \mathrm{~nm} \leq a_{\mathrm{g}} \leq 5 \mathrm{~nm}$. Hence, colloid attachment in primary minima is favored in the ATOP at $0.2 \mathrm{M}$ in these cases. For $2 \mathrm{~nm} \leq a_{\mathrm{g}} \leq 3 \mathrm{~nm}$, the detachment energy barriers are also reduced to be smaller than $1.5 \mathrm{kT}$ at $\leq 0.1 \mathrm{M}$. This indicates that these asperity radii not only favor attachment in primary minima at high ionic strengths but also benefit subsequent detachment upon reduction of solution ionic strengths.

Figure 5 shows DLVO interaction energy curves for interactions between a $1156 \mathrm{~nm}$ colloid and the rough surface in Figure 1a of different asperity radii along the $z$-axis at different ionic strengths. The energy barriers disappear for 2 $\mathrm{nm} \leq a_{\mathrm{g}} \leq 10 \mathrm{~nm}$ at $0.1 \mathrm{M}$ and for $2 \mathrm{~nm} \leq a_{\mathrm{g}} \leq 30 \mathrm{~nm}$ at 0.2 $\mathrm{M}$. Hence, colloid attachment in primary minimum is favored in the ATOP in these case. For $2 \mathrm{~nm} \leq a_{\mathrm{g}} \leq 10 \mathrm{~nm}$, the interaction energy decreases monotonically with increasing separation distance at 0.01 and $0.001 \mathrm{M}$, indicating that the colloid experiences repulsive force at all separation distances. Hence, the colloids attached atop of these asperities in primary minima at $\geq 0.1 \mathrm{M}$ will detach upon reduction of solution ionic strength. For $a_{\mathrm{g}}=0 \mathrm{~nm}$ and $a_{\mathrm{g}} \geq 20 \mathrm{~nm}$, the depth of the primary minimum increases with decreasing ionic strength and the potential interaction energy increases more rapidly from zero separation distance at lower ionic strength. Attachment in primary minima is thus irreversible to reduction of solution ionic strength in these cases. The results in Figure 5 reveal that more irreversible attachments occur at higher ionic strengths (e.g., $0.2 \mathrm{M}$ compared to $0.1 \mathrm{M}$ ), which provides plausible explanation for the observed hysteresis of colloid attachment and detachment during transients in solution chemistry. ${ }^{35}$

Interestingly, if the collector surface is assumed to be perfectly smooth, the calculated maximum energy barrier is much larger for the $1156 \mathrm{~nm}$ colloid than that for the $66 \mathrm{~nm}$ colloid at a given ionic strength. The large energy barriers essentially prevent the microsized colloid from being attached in primary minima even at $0.2 \mathrm{M}$. When surface roughness is 

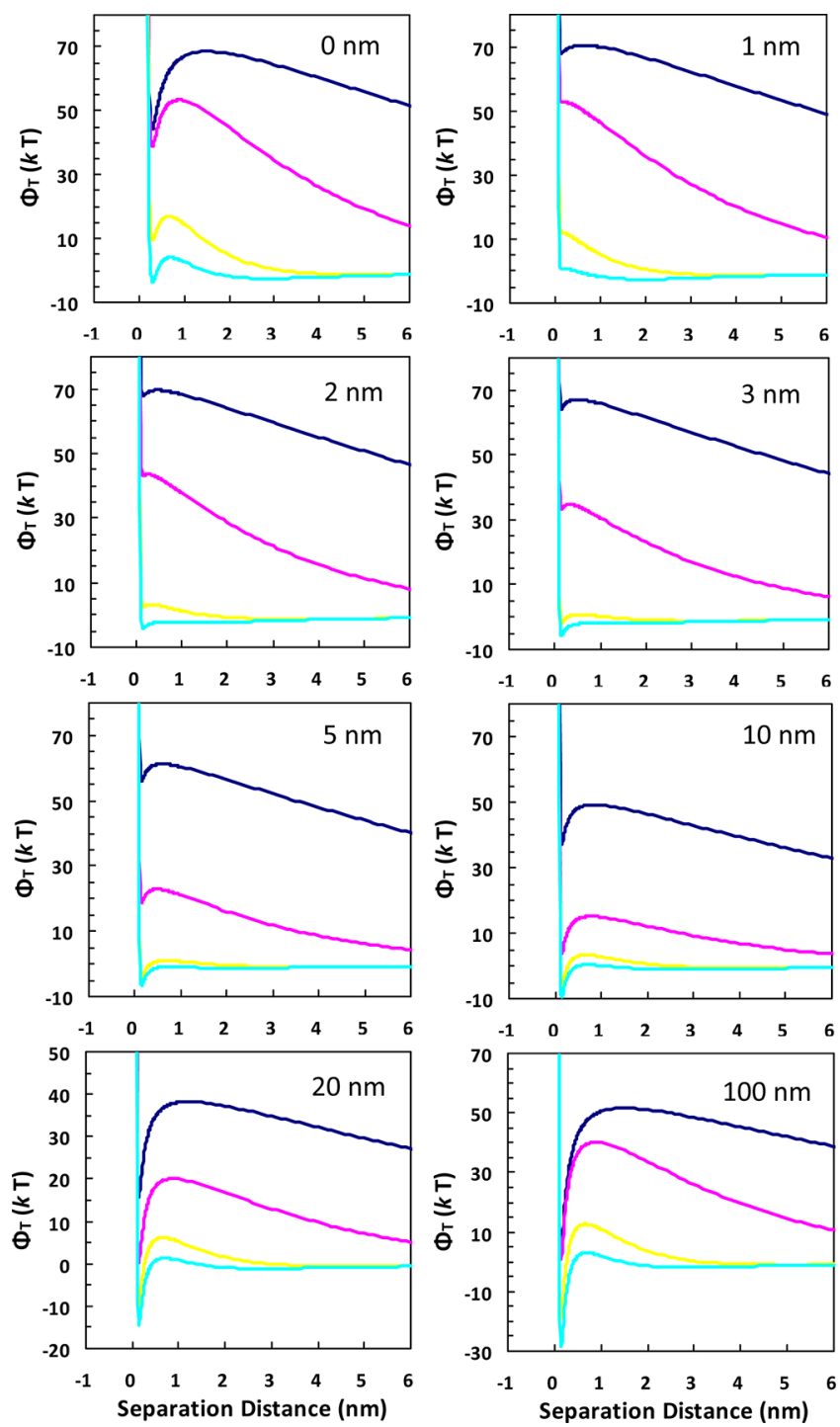

Figure 4. DLVO energy curves for interactions between the $66 \mathrm{~nm}$ colloid and rough surface in Figure la of different asperity radii along the $z$-axis at different ionic strengths (dark blue, $0.001 \mathrm{M}$; pink, 0.01 $\mathrm{M}$; yellow, 0.1 M; turquoise, $0.2 \mathrm{M}$ ).

considered, the range of asperity radii that cause absence of energy barrier in the ATOP is wider for the microsized colloid than the nanoparticle at a given ionic strength. This suggests that colloid attachment in primary minima could be more for the microsized colloid than for the nanoparticle under unfavorable conditions. Indeed, a number of column experiments ${ }^{13,26,28,36}$ show that the attachment efficiencies are larger for the microsized colloids than those for nanoparticles at low ionic strengths although classic colloid filtration theory (CFT) predicts that the attachment efficiencies for microsized colloids are many orders of magnitude smaller than those for nanoparticles. However, the aforementioned result is only valid in cases where colloid-nanoasperity interaction dominates attachment. Lager colloids may have lower attachment efficiencies if hydrodynamic shear is large enough to prevent them from being attached on asperities.

Figure 6 presents DLVO energy maps for interactions between the $66 \mathrm{~nm}$ colloid and rough configuration in Figure $1 \mathrm{~b}$ of $20 \mathrm{~nm}$ asperity radius on the $x-z$ plane at ionic strengths of $0.001,0.01,0.1$, and $0.2 \mathrm{M}$. The energy barrier is enhanced in
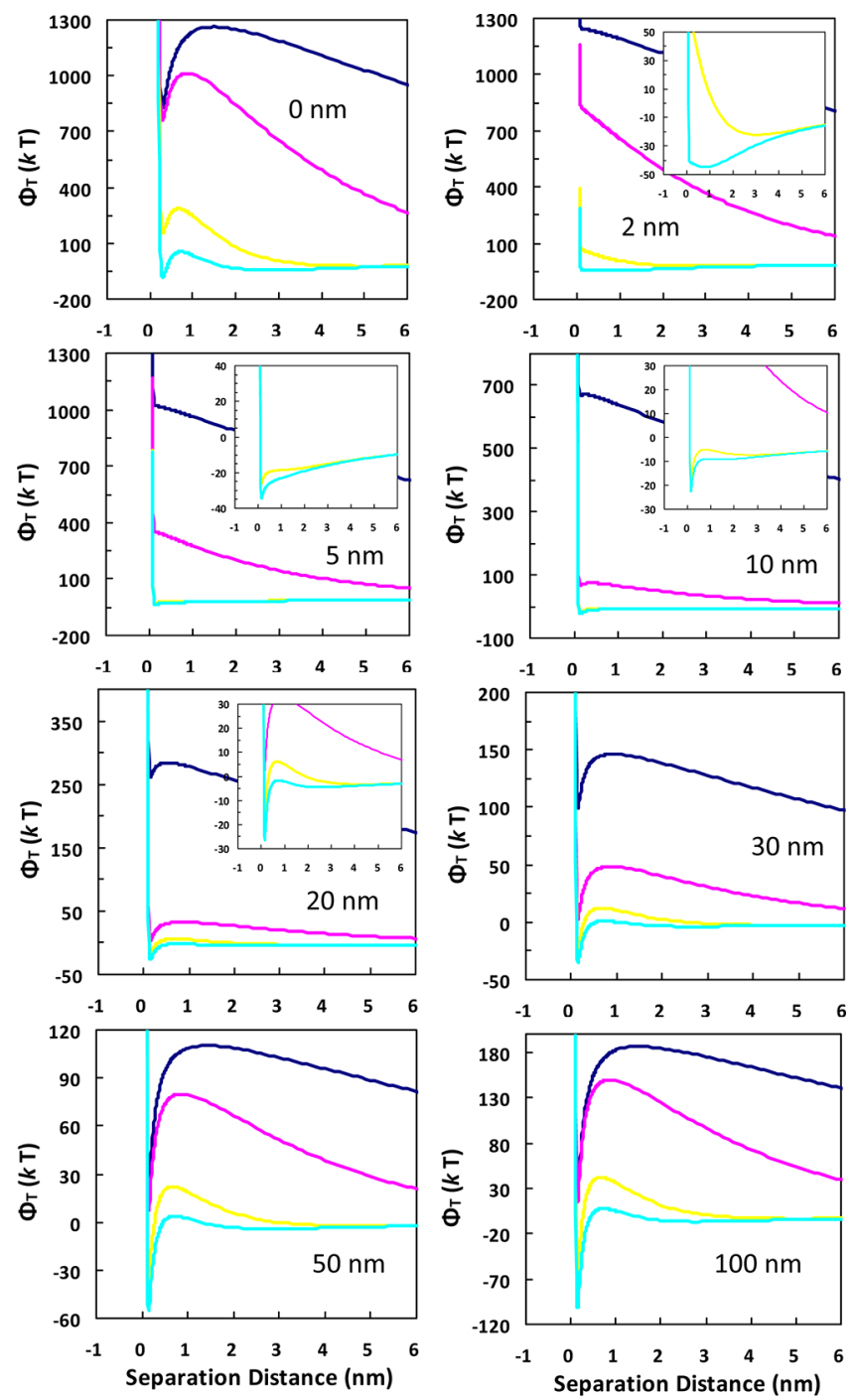

Figure 5. DLVO energy curves for interactions between a $1156 \mathrm{~nm}$ colloid and rough surface in Figure 1a of different asperity radii along the $z$-axis at different ionic strengths (dark blue, $0.001 \mathrm{M}$; pink, 0.01 $\mathrm{M}$; yellow, 0.1 M; turquoise, $0.2 \mathrm{M}$ ). Note the change in scale of the $y$ axes among the various graphs. Insets are replotted figures at a different axis scale to highlight the primary minimum depth.

both ASIDE and the region between asperities (denoted as BETWEEN). Therefore, attachment in primary minima is only favored on convex surfaces with small curvatures at high ionic strengths, as have been discussed previously. The enhancement of energy barrier in the ASIDE and BETWEEN is because of additional repulsion from the sidewalls. The sidewall interaction, however, also increases secondary minimum depth in the concave regions (i.e., ASIDE and BETWEEN) (see Figures $3 \mathrm{~b}$ and $6 \mathrm{~b}$ ). The impact of surface roughness on secondary minimum attachment is examined in detail in the following section.

It should be mentioned that this study used a high value of Hamaker constant $\left(1 \times 10^{-20} \mathrm{~J}\right)$ to calculate van der Waals force. The irreversible attachments in primary minima will be less at a given ionic strength if lower values of Hamaker constant are used. In addition, we adopted a value of $0.5 \mathrm{~nm}$ for the Born collision parameter, as have been used in previous studies. ${ }^{37,38}$ The irreversible attachments in primary minima 

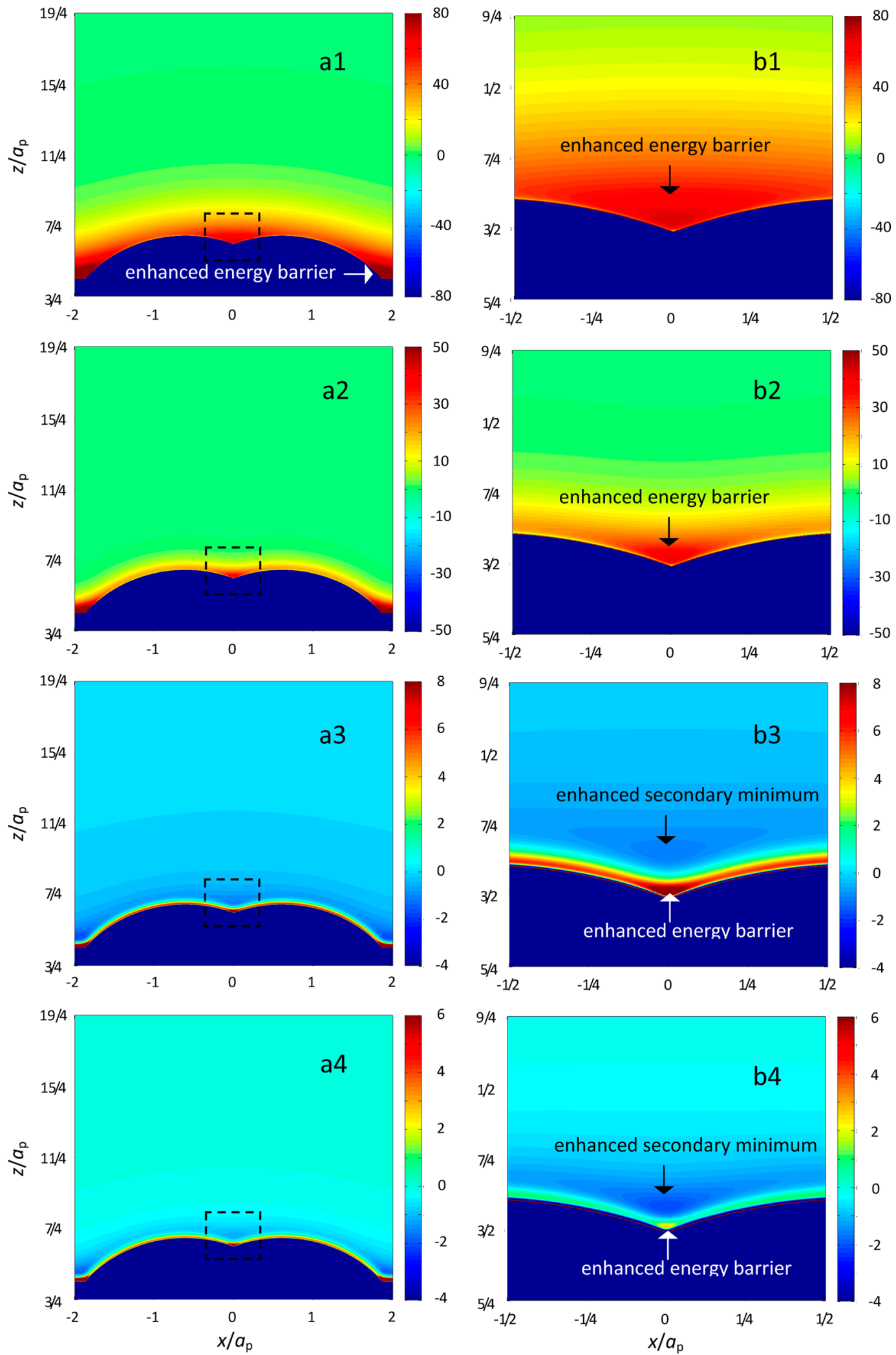

Figure 6. DLVO energy maps for interactions between the $66 \mathrm{~nm}$ colloid and rough configuration in Figure $1 \mathrm{~b}$ of $20 \mathrm{~nm}$ asperity radius on the $x-z$ plane at $y=0$ at different ionic strengths $(1,0.001 \mathrm{M} ; 2,0.01 \mathrm{M} ; 3,0.1 \mathrm{M} ; 4,0.2 \mathrm{M})$. All simulation conditions were consistent with those in Figure 2 except the interaction energy scales.

will be decreased and increased with increasing and decreasing the value of Born collision parameter, respectively.

3.2. Impact of Surface Roughness on Attachment in Secondary Minima. If the collector surface is assumed to be perfectly smooth (as done in the CFT), the secondary minima are commonly shallow wells in interaction energy curves under unfavorable conditions. For example, Figure 7 shows calculated secondary minimum depths for colloid-flat surface interactions 

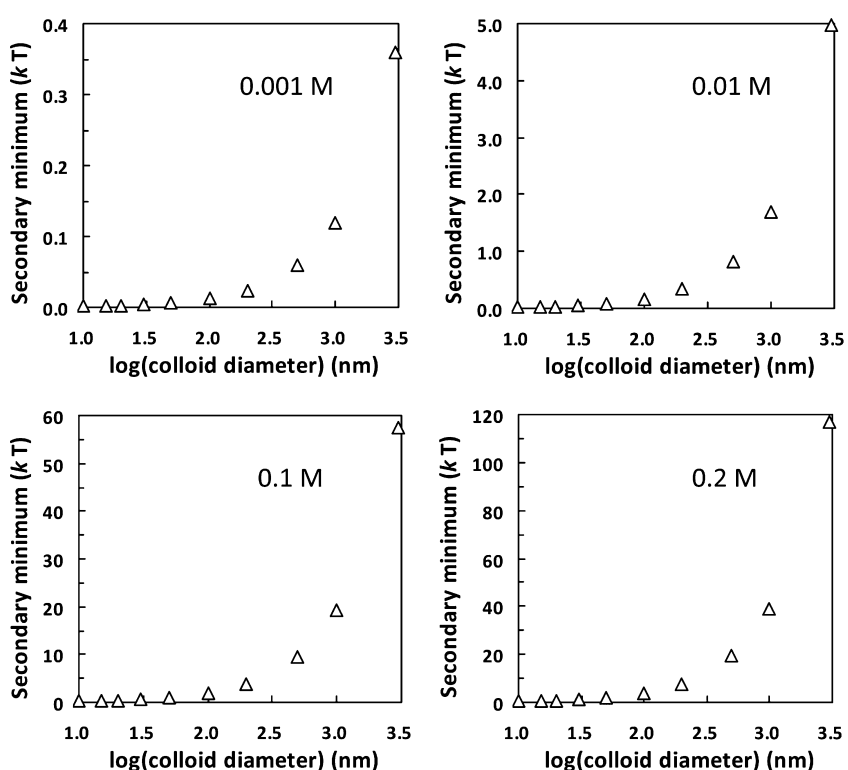

Figure 7. Calculated secondary minimum depths for interactions between a colloid and flat surface as a function of colloid diameter at different ionic strengths. Measured zeta potentials for $1156 \mathrm{~nm}$ colloids and sand grains from Shen et al. ${ }^{28}$ in Table S2 of the Supporting Information were used for calculation. Note the change in scale of the $y$-axes among the various graphs.

with different diameters at $0.001,0.01,0.1$, and $0.2 \mathrm{M}$. The calculated secondary minimum depths are very small for the nanoparticles even at $0.2 \mathrm{M}(<2.5 \mathrm{kT}$ for $\leq 100 \mathrm{~nm}$ colloids $)$ and for the large colloids at low ionic strengths (e.g., $<2 k T$ for $1000 \mathrm{~nm}$ colloid at $\leq 0.01 \mathrm{M}$ ionic strengths). Thus, attachment in secondary minima is not expected for nanoparticles under unfavorable conditions and for large colloids at low ionic strengths because the colloids can easily escape from the shallow wells by Brownian diffusion. However, attachment in secondary minima is frequently reported in experimental studies under these conditions. For example, Shen et al. ${ }^{28}$ showed attachment in secondary minimum for a $30 \mathrm{~nm}$ colloid in sand columns at 0.001, 0.01, 0.1, and 0.2 M. Syngouna and Chrysikopoulos $^{39}$ reported attachment in secondary minima for bacteriophage MS2 and $\varphi$ X174 and Escherichia coli in sand columns at $0.002 \mathrm{M}$.

The AFM images show that the surfaces of sand grains are very rough. ${ }^{13,14}$ Particularly, Shen et al. ${ }^{13}$ showed that the average and maximum roughness for sand measured by AFM reach 293 and $2418 \mathrm{~nm}$, respectively. Even for relatively smooth glass bead surfaces, the average and maximum roughness reach 80 and $627 \mathrm{~nm}$, respectively. ${ }^{13}$ Figures $3 \mathrm{~b}$ and Figure $6 \mathrm{~b}$ show that the presence of surface roughness enhances the secondary minimum energy in the ASIDE and BETWEEN (i.e., concave regions). Figure 8 presents calculated largest secondary minimum energy for interactions between the $66 \mathrm{~nm}$ colloid and rough surface in Figure 1c in threedimensional space as a function of asperity radii at $0.2 \mathrm{M}$. The largest secondary minimum depth increases with increasing asperity radii and has reached three times that for the colloidflat surface interaction within the range of asperity radii considered. Hence, the $66 \mathrm{~nm}$ colloid can be attached stably at the sites with secondary minimum energies significantly greater than $1.5 \mathrm{kT}$.

Despite colloid attachment in secondary minima being recognized by more and more studies, ${ }^{4,17,18,40}$ this attachment

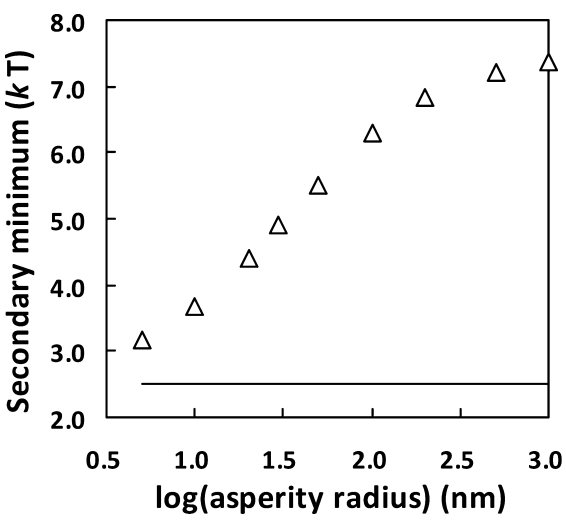

Figure 8. Largest secondary minimum depth (triangle) for interaction between the $66 \mathrm{~nm}$ colloid and the rough surface in Figure 1c in threedimensional space as a function of asperity radii at $0.2 \mathrm{M}$. The calculated secondary minimum depth for interaction between the 66 $\mathrm{nm}$ colloid and the flat surface (line) is shown for comparison. Measured zeta potentials for the $66 \mathrm{~nm}$ colloid for the sand grain from Shen et al. ${ }^{28}$ in Table S2 of the Supporting Information were used for calculation.

is regarded to occur only in stagnation point region of a porous medium and the colloids at other locations via secondary minimum association will translate and rotate along collector surface until reach the stagnation point region due to hydrodynamic shear. This view, however, is also based on the assumption that the collector surface is perfectly smooth. For interactions of colloids with rough collector surfaces, tangential forces are present which may balance the hydrodynamic shear and prevent the translation and rotation. To test the hypothesis, we calculated maps of the component of DLVO interaction force along $x$-direction $\left(F_{\mathrm{A}}\right)$ normalized by a hydrodynamic drag force $\left(F_{\mathrm{D}}\right)$ for interactions between the $66 \mathrm{~nm}$ colloid and rough configuration in Figure 1a of $100 \mathrm{~nm}$ asperity radius on the first quadrant of $x-z$ plane at different ionic strengths (Figure 9a). The equation used to calculate $F_{\mathrm{A}}$ is written as

$$
F_{\mathrm{A}}=-\left(\frac{\mathrm{d} \Phi_{\mathrm{pg}}^{\mathrm{vdw}}}{\mathrm{d} h}+\frac{\mathrm{d} \Phi_{\mathrm{pg}}^{\mathrm{dl}}}{\mathrm{d} h}\right) \sin \alpha
$$

where $\mathrm{d} \Phi_{\mathrm{pg}}^{\mathrm{vdw}} / \mathrm{d} h$ and $\mathrm{d} \Phi_{\mathrm{pg}}^{\mathrm{dl}} / \mathrm{d} h$ represent derivatives of $\Phi_{\mathrm{pg}}^{\mathrm{vdw}}$ and $\Phi_{\mathrm{pg}}^{\mathrm{dl}}$ with respect to $h$, respectively; $a$ is the angle by rotating the line through both centers of the colloid and the asperity counterclockwise on the $x-z$ plane in Figure 1a until it coincides with the $z$-axis. The value of $F_{\mathrm{D}}$ is calculated using the equation

$$
F_{\mathrm{D}}=3 \pi \mu d_{\mathrm{p}} U
$$

where $\mu$ is viscosity of water, $d_{\mathrm{p}}$ is colloid diameter, and $U$ is flow velocity. A typical rate of groundwater flow (i.e., $4 \mathrm{~cm}$ / day) was chosen for $U$, and the direction of $U$ was assumed to be along the positive $x$-direction of the coordinate system in Figure 1a. The positive and negative values of $F_{\mathrm{A}} / F_{\mathrm{D}}$ in Figure 9a represent the components of DLVO interaction force along positive (i.e., repulsion) and negative (i.e., attraction) $x$ directions, respectively. The DLVO interaction energy maps were also shown in Figure $9 \mathrm{~b}$ to denote the locations of secondary minima.

A comparison of Figure 9a with Figure 9b shows that the components of interaction forces along the $x$-direction are attractive in the region down gradient of the secondary 

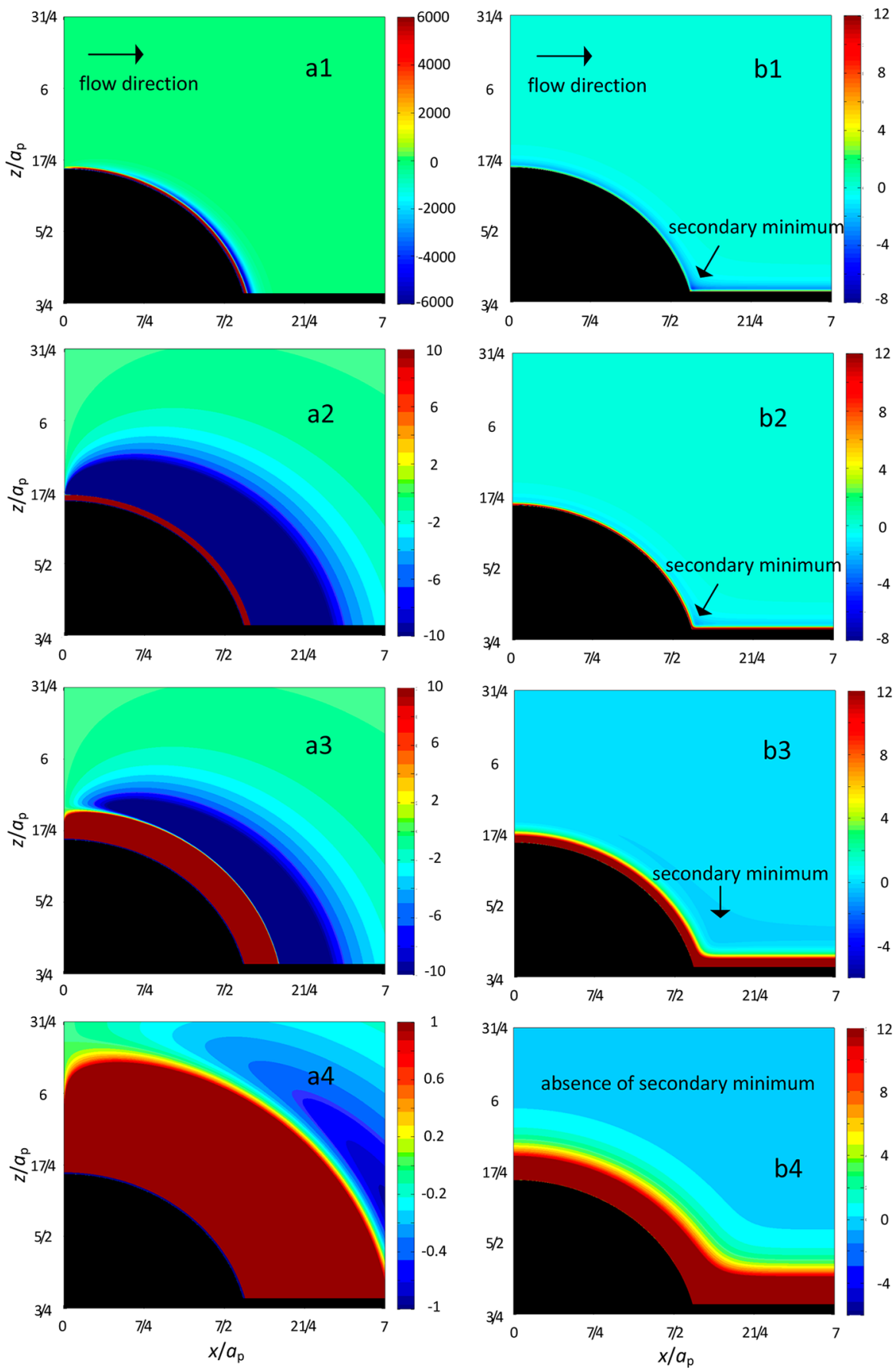

Figure 9. Maps of (a) components of DLVO interaction forces along $x$-direction $\left(F_{\mathrm{A}}\right)$ normalized by a hydrodynamic drag force $\left(F_{\mathrm{D}}\right)$ and $(\mathrm{b})$ DLVO interaction energy for the $66 \mathrm{~nm}$ colloid interacting with the rough configuration in Figure 1a of $100 \mathrm{~nm}$ asperity radius on the first quadrant of the $x-z$ plane at $y=0$ at different ionic strengths $(1,0.2 \mathrm{M} ; 2,0.1 \mathrm{M} ; 3,0.01 \mathrm{M} ; 4,0.001 \mathrm{M})$. The dark regions indicate the locations where the interaction energy could not be determined due to physical overlap between the colloid and rough surfaces. In (a), positive and negative values represent the component of DLVO interaction force along positive and negative $x$-directions, respectively. In (b), the scale bar to the right of each plot represents the interaction energy in units of $k T$. $F_{\mathrm{D}}$ is calculated as $3 \pi \mu d_{\mathrm{p}} U$, where $\mu$ is viscosity of water, $d_{\mathrm{p}}$ is colloid diameter, and $U$ is relatively velocity of the fluid with respect to the particle, taken as $4 \mathrm{~cm} /$ day.

minimum region with respect to the flow direction [i.e., the blue area on the right of red bar in Figure 9a], and they are significantly larger than the values of $F_{\mathrm{D}}$ at $\geq 0.01 \mathrm{M}$. Particularly, the maximum value of $F_{\mathrm{A}}$ in the region down gradient of the secondary minima is about $6 \times 10^{3}$ times larger than the value of $F_{D}$ at $0.2 \mathrm{M}$. Therefore, the colloids at secondary minima cannot be swept away by hydrodynamic shear due to the tangential attractive force at $\geq 0.01 \mathrm{M}$. Note that the actual hydrodynamic forces acting on the colloid at secondary minima are much smaller than the $F_{\mathrm{D}}$ obtained using eq 2 because of the shielding effect by the asperity and the wall effect from the collector surface. The results of Figure 9 theoretically explain the microscopic observations ${ }^{35,41}$ that attachment in secondary minima was not restricted to graingrain contacts of a porous medium but the attached colloids were almost evenly distributed on open collector surfaces when 
solution ionic strength was high $(\geq 0.01 \mathrm{M})$. Figure 9 also indicates that the attached colloids in shear flow (e.g., impinging jet systems) are not necessarily at primary minima but could also be associated with surfaces at secondary minima. Kalasin and Santore ${ }^{42}$ and Liu et al. ${ }^{43}$ have provided microscopic evidence for secondary minimum attachment of microparticles in shear flow. In addition, the column-scale experiments ${ }^{13}$ show that surface roughness increases attachment in secondary minima.

We only considered a typical rate of groundwater flow to calculate $F_{D}$ in Figure 9. Because the value of $F_{D}$ increases with increasing flow rate, the ionic strengths at which secondary minima attachment can be located on open collector surfaces will be higher for larger flow velocities. Figure S2 of the Supporting Information presents maps of $F_{\mathrm{A}} / F_{\mathrm{D}}$ for interactions between a $500 \mathrm{~nm}$ colloid and rough configuration in Figure 1a of $100 \mathrm{~nm}$ asperity radius on the first quadrant of the $x-z$ plane at different ionic strengths. Results show that, similar to the $66 \mathrm{~nm}$ colloid, the $500 \mathrm{~nm}$ colloid can also be attached in secondary minima on open collector surfaces at $\geq 0.01 \mathrm{M}$. However, because the particle size effects are more significant for hydrodynamic drag force than DLVO force, the values of $F_{\mathrm{A}} / F_{\mathrm{D}}$ are smaller for the $500 \mathrm{~nm}$ colloid than for the $66 \mathrm{~nm}$ colloid at a given ionic strength.

\section{DISCUSSION}

Considerable theoretical studies ${ }^{7,9-15,22,27,29}$ illustrated that surface roughness reduces interaction energy barrier and hence increases colloid attachment in primary minima. The detachment behavior of the excessively attached colloids, however, was not examined. Results of this study show that although colloids are favored to be attached atop of nanoasperities in primary minima at high ionic strengths, a fraction of the attached colloids are also facilitated to detach upon reduction of solution ionic strengths. The fraction of irreversible attachment in primary minima is larger at higher ionic strengths.

Whereas colloid attachment in secondary minima is being recognized by more and more studies, $4,17,18,37,38,41,43-45$ the argument about the role of secondary energy minima in colloid attachment has not stopped mainly because of two reasons. First, the secondary minima are regarded as shallow wells in the DLVO interaction energy curves and colloids at the wells could easily escape by Brownian motion. Second, the secondary minimum is located at a distance from the collector surface; thus, the colloids at the secondary minima suffer from hydrodynamic shear in fluid and could be swept away easily. By using DLVO interaction energy map, our study shows that the presence of surface roughness can (i) increases secondary minimum depths significantly in local areas (e.g., ASIDE and BETWEEN) and (ii) provides tangential adhesive forces to prevent colloids from being swept away by hydrodynamic shear. Therefore, we theoretically justify the presence of attachment in secondary minima under unfavorable chemical conditions.

The surfaces of natural collectors all contain some degree of physical nonuniformity at various scales. ${ }^{15}$ Interestingly, even if only convex asperities exist on a rough surface, the interactions of a colloid with concave-like surfaces (e.g., ASIDE and BETWEEN) are commonly dominant over the interactions of the colloid with convex-like surfaces (i.e., ATOP) (see Figure 10). Whereas the convex-like surfaces decreases energy barrier and favor the colloid reaching primary minima, ${ }^{11}$ the hydrodynamic shear is significant and both the force and the
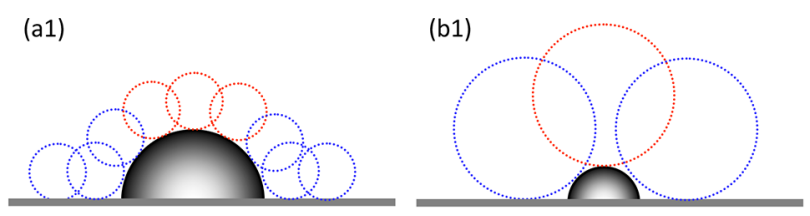

(a2)
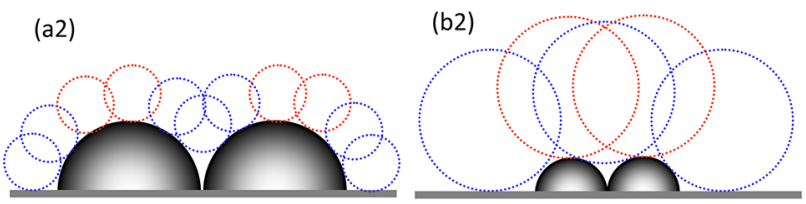

(a3)
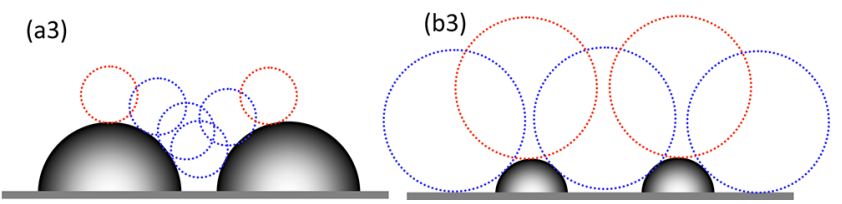

Figure 10. Schematics used to demonstrate interaction morphologies influenced by the ratio of colloid size to asperity size and by the distance between asperities. The dotted circles represent a colloid at different locations of a rough surface. The red and blue circles represent colloids interacting with convex-like and concave-like surfaces, respectively.

level arm of adhesive torque are decreased at these locations. ${ }^{16,46-48}$ In contrast, although the concave surfaces increase energy barriers, they also increase secondary minimum depths and colloids at these locations experience tangential attractions and are shielded from hydrodynamic shear. Therefore, the presence of surface roughness is likely more favorable for attachment in secondary minima than in primary minima. Indeed, by using AFM, Bowen and Doneva ${ }^{49}$ showed that although the repulsion between a colloid probe and a membrane at peaks on the membrane surface is greatly reduced compared to that in valleys, adhesion of the colloid probe is lower at the peaks on the membrane surface than in the valleys with the difference increasing with decreasing salt concentration. The sand column experiments ${ }^{13,28,37,38}$ also showed that the secondary minimum plays a dominant role in colloid attachment under unfavorable conditions.

For the colloid-flat surface interaction, smaller particles have lower secondary minimum depths and are expected to have less secondary minimum attachments. ${ }^{37,38}$ However, if surface roughness is present, smaller colloids could access more concave regions for a given rough surface geometry (see Figure 10) and thus are more favorable for attachment in secondary minima. Darbha et al. ${ }^{50,51}$ found that the influence of surface roughness on colloid retention is more significant for smaller colloids under unfavorable conditions. Shen et al. ${ }^{28}$ showed that secondary minima play a dominant role in colloid attachment even for a $30 \mathrm{~nm}$ nanoparticle. Chen et al. ${ }^{52}$ demonstrated that larger rough asperities (causing larger concave regions) result in greater colloid attachment. In addition to the ratio of colloid size to asperity size, other roughness properties such as the distance between asperities also influence colloidal access to concave regions (see Figure 10) and accordingly influence the attachment in secondary minima.

The AFM images $7,13,14,19,50,51$ show that the roughness of natural collector surfaces (e.g., sand) is much more complex compared to the model rough surfaces used in this study. Various concave-like surfaces are present due to the fractal nature of the roughness. Obviously, the interactions of a colloid 
with the different concave-like surfaces result in a distribution of secondary minimum depths. For given chemical and hydrodynamic conditions, the concave sites that could cause colloids to be attached stably in secondary minima are limited in a porous medium. For a concave site that is favorable for the attachment, the number of colloids can be filled is limited, depending on the morphology of the concave site and the size of colloid. Therefore, colloid attachment in secondary minima is time dependent (i.e., blocking) and colloid concentration dependent, as observed in experiments. ${ }^{53,54}$

Secondary minimum energy, surface roughness, and surface charge heterogeneity are frequently regarded as the main factors causing the discrepancies between experimental deposition rates and CFT predictions. ${ }^{4,17,18,42,55,56}$ Results of this study indicate that the impacts of secondary minimum energy and surface roughness on colloid attachment are coupled. The influence of surface charge heterogeneity, however, is not considered in this study. In geologic environments, the charge heterogeneity is common on surfaces of natural aquifer materials. Bendersky and Davis ${ }^{22}$ showed that the coupled influence of surface roughness and charge heterogeneity enhances reduction of energy barrier and accordingly increases attachment in primary minima. The coupled influence of surface roughness and charge heterogeneity on attachment in secondary minima is currently a topic of ongoing investigation but is beyond the scope of this study.

\section{CONCLUSIONS}

This study first examined effects of surface roughness on attachment in and subsequent detachment from primary minima using DLVO interaction energy map and curve. Results show that while colloids are favored to be deposited atop of the nanoasperities via primary minimum association at high ionic strengths (e.g, $\geq 0.1 \mathrm{M}$ in this study), a fraction of the attached colloids are also facilitated to be detached from primary minimum upon reduction of solution ionic strength. The fraction of irreversible attachment in primary minima is larger at higher ionic strengths. This theoretically explains the observed hysteresis of colloid attachment and detachment during transients in solution chemistry in the literature.

This study further highlights the significance of surface roughness in colloid attachment in secondary minima. It was shown that secondary minimum depths can be increased significantly in concave regions. Moreover, through comparing the tangential attractive forces from asperities and hydrodynamic drag forces in three-dimensional space, we showed that the attachment in secondary minima can be located on open collector surfaces of a porous medium. This challenges the usual belief that attachment in secondary minima only occurs in stagnation point regions of the porous medium and is absent in shear flow systems such as parallel plate flow chamber and impinging jet apparatus. Our study implies that the presence of surface roughness is more favorable for colloid attachment in secondary minima than in primary minima under unfavorable chemical conditions.

\section{ASSOCIATED CONTENT}

\section{S Supporting Information}

The reasons about why the surface element integration cannot accurately estimate colloid-rough surface interaction, the maps of tangential DLVO forces normalized by a hydrodynamic drag force for the $500 \mathrm{~nm}$ colloid interacting with the rough configuration in Figure 1a of $100 \mathrm{~nm}$ asperity radius, the separation distances between a colloid and a hemisphere or the flat surface for the rough models in Figure 1, and the zeta potentials for the colloids and collector. This material is available free of charge via the Internet at http://pubs.acs.org.

\section{AUTHOR INFORMATION}

\section{Corresponding Author}

*Phone +86 15011558633; Fax +86 1062733596; e-mail chongyang.shen@gmail.com (C.S.). Phone +86 1062732963; Fax +86 1062733596; e-mail yfhuang@cau.edu.cn (Y.H.).

\section{Notes}

The authors declare no competing financial interest.

\section{ACKNOWLEDGMENTS}

We acknowledge the financial support provided by the Beijing Natural Science Foundation (no. 6123034), National Natural Science Foundation of China (no. 41271009, 40901109), National Key Technology R\&D Program (no. 2012BAD05B02), Specialized Research Fund for the Doctoral Program of Higher Education (no. 20090008120041), and the Chinese Universities Scientific Fund (no. 2011JS165, no. 2012QJ163).

\section{REFERENCES}

(1) Derjaguin, B. V.; Landau, L. Theory of the stability of strongly charged lyophobic sols and of the adhesion of strongly charged particles in solutions of electrolytes. Acta Physicochim. USSR 1941, 14, $633-652$.

(2) Verwey, E. J. W.; Overbeek, J. Th. G. Theory of the Stability of Lyophobic Colloids; Elsevier: Amsterdam, 1948.

(3) Swanton, S. W. Modeling colloid transport in groundwater: The prediction of colloid stability and retention behavior. Adv. Colloid Interface Sci. 1995, 54, 129-208.

(4) Ryan, J. N.; Elimelech, M. Colloid mobilization and transport in groundwater. Colloids Surf., A 1996, 107, 1-56.

(5) Adamczyk, Z.; Weronski, P. Application of the DLVO theory for particle deposition problems. Adv. Colloid Interface Sci. 1999, 83, 137226.

(6) Grasso, D.; Subramaniam, K.; Butkus, M.; Strevett, K.; Bergendahl, J. A review of non-DLVO interactions in environemntal colloidal systems. Rev. Environ. Sci. Biotechnol. 2002, 1, 17-38.

(7) Hoek, E. M. V.; Bhattacharjee, S.; Elimelech, M. Effect of membrane surface roughness on colloid-membrane DLVO interactions. Langmuir 2003, 19, 4836-4847.

(8) Kemps, J. A. L.; Bhattacharjee, S. Interactions between a solid spherical particle and a chemically heterogeneous planar substrate. Langmuir 2005, 21, 11710-11721.

(9) Bhattacharjee, S.; Ko, C.-H.; Elimelech, M. DLVO interaction between rough surfaces. Langmuir 1998, 14, 3365-3375.

(10) Hoek, E. M. V.; Agarwal, G. K. Extended DLVO interactions between spherical particles and rough surfaces. J. Colloid Interface Sci. 2006, 298, 50-58.

(11) Huang, X.; Bhattacharjee, S.; Hoek, E. M. V. Is surface roughness a "scapegoat" or a primary factor when defining particlesubstrate interactions? Langmuir 2010, 26, 2528-2537.

(12) Henry, C.; Minier, J.-P.; Lefevre, G.; Hurisse, O. Numerical study on the deposition rate of hematite particle on polypropylene walls: role of surface roughness. Langmuir 2011, 27, 3603-4612.

(13) Shen, C.; Li, B.; Wang, C.; Huang, Y.; Jin, Y. Surface roughness effect on deposition of nano- and micro-sized colloids in saturated columns at different solution ionic strengths. Vadose Zone J. 2011, 10, 1071-1081.

(14) Shen, C.; Wang, L.-P.; Li, B.; Huang, Y.; Jin, Y. Role of surface roughness in chemcial detachment of colloids deposited at primary energy minima. Vadose Zone J. 2012, DOI: 10.2136/vzj2011.0057. 
(15) Suresh, L.; Walz, J. Y. Effect of surface roughness on the interaction energy between a colloidal sphere and a flat plate. J. Colloid Interface Sci. 1996, 183, 199-213.

(16) Eichenlaub, S.; Gelb, A.; Beaudoin, S. Roughness models for particle adhesion. J. Colloid Interface Sci. 2004, 280, 289-298.

(17) Elimelech, M.; O'Melia, C. R. Kinetics of deposition of colloidal particles in porous media. Environ. Sci. Technol. 1990, 24 (10), 15281536.

(18) Elimelech, M.; O’Melia, C. R. Effect of particle size on collision efficiency in the deposition of Brownian particles with electrostatic energy barriers. Langmuir 1990, 6 (6), 1153-1163.

(19) Shellenberger, K.; Logan, B. E. Effect of molecular scale roughness of glass beads on colloidal and bacterial deposition. Environ. Sci. Technol. 2002, 36, 184-189.

(20) Jaiswal, R. P.; Kumar, G.; Kilroy, G. M.; Beaudoin, S. P. Modeling and validation of the van der Waals forces during the adhesion of nanoscale objects to rough surfaces: a detailed description. Langmuir 2009, 25 (18), 10612-10623.

(21) Bhattacharjee, S.; Elimelech, M. Surface element integration: a novel technique for evaluation of DLVO interaction between a particle and a flat plate. J. Colloid Interface Sci. 1997, 193, 273-285.

(22) Bendersky, M.; Davis, J. M. DLVO interaction of colloidal particles with topographically and chemically heterogeneous surfaces. J. Colloid Interface Sci. 2011, 353, 87-97.

(23) Hamaker, H. C. The London-van der Waals attraction between spherical particles. Physica 1937, 4, 1058-1072.

(24) Hogg, R.; Healy, T. W.; Fuerstenau, D. W. Mutual coagulation of colloidal dispersions. Trans. Faraday Soc. 1966, 62, 1638-1651.

(25) Duffadar, R. D.; Davis, J. M. Interaction of micrometer-scale particles with nanotextured surfaces in shear flow. J. Colloid Interface Sci. 2007, 308, 20-29.

(26) Shen, C.; Huang, Y.; Li, B.; Jin, Y. Effects of solution chemistry on straining of colloids in porous media under unfavorable conditions. Water Resour. Res. 2008, 44, W05419 DOI: 10.1029/2007WR006580.

(27) Shen, C.; Lazouskaya, V.; Zhang, H.; Wang, F.; Li, B.; Jin, Y.; Huang, Y. Theoretical and experimental investigation of detachment of colloids from rough collector surfaces. Colloids Surf., A 2012, DOI: $10.1016 /$ j.colsurfa.2012.06.025.

(28) Shen, C.; Li, B.; Huang, Y.; Jin, Y. Kinetics of coupled primaryand secondary-minimum deposition of colloids under unfavorable chemical conditions. Environ. Sci. Technol. 2007, 41, 6976-6982.

(29) Shen, C.; Lazouskaya, V.; Jin, Y.; Li, B.; Ma, Z.; Zheng, W.; Huang, Y. Coupled factors influencing detachment of nano- and micro-sized particles from primary minima. J. Contam. Hydrol. 2012, 134-135, 1-11.

(30) Camesano, T. A.; Logan, B. E. Probing bacterial electrosteric interactions using atomic force microscopy. Environ. Sci. Technol. 2000, 34, 3354-3362.

(31) Considine, R. F.; Drummond, C. J. Surface roughness and surface force measurement: A comparison of electrostatic potentials derived from atomic force microscopy and electrophoretic mobility measurements. Langmuir 2001, 17, 7777-7783.

(32) Brant, J. A.; Childress, A. E. Membrane-colloid interactions: Comparison of extended DLVO predictions with AFM force measurements. Environ. Eng. Sci. 2002, 19 (6), 413-427.

(33) Assemi, S.; Nalaskowski, J.; Johnson, W. P. Direct force measurements between carboxylate-modified latex microspheres and glass using atomic force microscopy. Colloids Surf., A 2006, 286, 7077.

(34) Acuna, S. M.; Toledo, P. G. Short-range forces between glass surfaces in aqueous solutions. Langmuir 2008, 24, 4881-4887.

(35) Torkzaban, S.; Kim, H. N.; Simunek, J.; Bradford, S. A. Hystersis of colloid retention and release in saturated porous media during transients in solution chemistry. Environ. Sci. Technol. 2010, 44, 16621669.

(36) Shen, C.; Huang, Y.; Li, B.; Jin, Y. Predicting attachment efficiency of colloid deposition under unfavorable attachment conditions. Water Resour. Res. 2010, 46, W11526 DOI: 10.1029/ 2010WR009218.
(37) Hahn, M. W.; O'Melia, C. R. Deposition and reentrainment of Brownian particles in porous media under unfavorable chemical conditions: Some concepts and applications. Environ. Sci. Technol. 2004, 38, 210-220.

(38) Hahn, M. W.; Abadzic, D.; O'Melia, C. R. Aquasols: On the role of secondary minima. Environ. Sci. Technol. 2004, 38, 5929-5924.

(39) Syngouna, V. I.; Chrysikopoulos, C. V. Transport of biocolloids in water saturated columns packed with sand: Effect of grain size and pore water velocity. J. Contam. Hydrol. 2011, 126, 301-314.

(40) Rajagopalan, R.; Tien, C. Trajectory analysis of deep-bed filtration with the sphere-in-cell porous medel. AIChE J. 1976, 2 (3), 523-533.

(41) Kuznar, Z. A.; Elimelech, M. Direct microscopic observation of particle deposition in porous media: Role of the secondary energy minimum. Colloids Surf., A 2007, 294, 156-162.

(42) Kalasin, S.; Santore, M. M. Sustained rolling of microparticles in shear flow over an electrostatically patchy surface. Langmuir 2010, 26, 2317-2324.

(43) Liu, Y.; Janjaroen, D.; Kuhlenschmidt, M. S.; Kuhlenschmidt, T. B.; Nguyen, T. H. Deposition of Cryptosporidium parvum oocysts on natural organic matter surfaces: Microscopic evidence for secondary minimum deposition in a radial stagnation point flow cell. Langmuir 2009, 25, 1594-1605.

(44) Redman, J. A.; Walker, S. L.; Elimelech, M. Bacterial adhesion and transport in porous media: role of the secondary energy minimum. Environ. Sci. Technol. 2004, 38, 1777-1785.

(45) Tufenkji, N.; Elimelech, M. Breakdown of colloid filtration theory: Role of the secondary energy minimum and surface charge heterogeneities. Langmuir 2005, 21, 841-852.

(46) Katainen, J.; Paajanen, M.; Ahtola, E.; Pore, V.; Lahtinen, J. Adhesion as an interplay between particle size and surface roughness. J. Colloid Interface Sci. 2006, 304, 524-529.

(47) Cooper, K.; Gupta, A.; Beaudoin, S. Substrate morphology and particle adhesion in reacting systems. J. Colloid Interface Sci. 2000, 228, 213-219.

(48) Cooper, K.; Gupta, A.; Beaudoin, S. Simulation of the adhesion of particles to surfaces. J. Colloid Interface Sci. 2001, 234, 284-292.

(49) Bowen, W. R.; Doneva, T. A. Atomic force microscopy studies of membranes: Effect of surface roughness on double-layer interactions and particle adhesion. J. Colloid Interface Sci. 2000, 229, 544-549.

(50) Darbha, G. K.; Schafer, T.; Heberling, F.; Luttge, A.; Fischer, C. Retention of latex colloids on calcite as a function of surface roughness and topography. Langmuir 2010, 26, 4743-4752.

(51) Darbha, G. K.; Fischer, C.; Michler, A.; Luetzenkirchen, J.; Schafer, T.; Heberling, F.; Schild, D. Deposition of latex colloids at rough mineral surfaces: An analogue study using nanopatterned surfaces. Langmuir 2012, 28, 6606-6617.

(52) Chen, G.; Bedi, R.; Yan, Y. S.; Walker, L. Initial colloid deposition on bare and zeolite-coated stainless steel and aluminum: influence of surface roughness. Langmuir 2010, 26, 12605-12613.

(53) Bradford, S. A.; Bettahar, M. Concentration dependent transport of colloids in saturated porous media. J. Contam. Hydrol. 2006, 82, 99-117.

(54) Bradford, S. A.; Kim, H. N.; Haznedaroglu, B. Z.; Torkzaban, S.; Walker, S. L. Coupled factors influencing concentration-dependent colloid transport and retention in saturated porous media. Environ. Sci. Technol. 2009, 43, 6996-7002.

(55) Duffadar, R.; Kalasin, S.; Davis, J. M.; Santore, M. M. The impact of nanoscale chemical features on micron-scale adhesion: Crossover from heterogeneity- dominated to mean-field behavior. J. Colloid Interface Sci. 2009, 337, 396-407.

(56) Kalasin, S.; Dabkowski, J.; Nusslein, K.; Santore, M. M. The role of nano-scale heterogeneous electrostatic interactions in initial bacterial adhesion from flow: A case study with Staphylococcus aureus. Colloids Surf., B 2010, 76, 489-495. 